\title{
Interação com artefatos e personagens artificiais em mundos virtuais
}

\author{
Fabiana Santiago Sgobbi ${ }^{1}$, Felipe Becker Nunes ${ }^{1}$, Andreia Solange Bos ${ }^{2}$, Giliane \\ Bernardi $^{2}$, Liane Margarida Rockenbach Tarouco ${ }^{1}$ \\ ${ }^{1}$ Universidade Federal do Rio Grande do Sul (UFRGS) \\ ${ }^{2}$ Universidade Federal de Santa Maria (UFSM) \\ santiagofabiana@hotmail.com, nunesfb, andreia.bos@gmail.com, \\ gilianedinf.ufsm.br, liane@penta.ufrgs.br
}

\begin{abstract}
This paper presents a proposal for the organization of immersive environments to provide active learning activities using virtual artifacts and supported by Agents. The environment was used OpenSim, and it in addition to, artifacts that exhibit behavior simulating real experiments and virtual agents were deployed. To extend the flexibility of the interactions between the student, through his avatar and the NPC, a connection of this with a chatterbot, whose knowledge base was prepared to provide relevant answers to the context of the experiment was established. The agent also performs the tracking of student activities in the virtual world, exporting the collected data to external systems.
\end{abstract}

Resumo: Este artigo apresenta uma proposta de organização de ambientes imersivos para ensejar atividades de aprendizagem ativa utilizando artefatos virtuais e com o apoio de agentes. $O$ ambiente utilizado foi o OpenSim, e nele, além dos artefatos que exibem comportamento simulando experimentos reais, foram implantados agentes. Para ampliar a flexibilidade das interações entre o estudante, através de seu avatar, e o NPC, foi estabelecida uma conexão deste com um chatterbot, cuja base de conhecimento foi preparada para oferecer respostas relevantes ao contexto do experimento realizado. $O$ agente também realiza o rastreamento das atividades do estudante no mundo virtual, exportando os dados coletados para sistemas externos.

\section{Interatividade em ambientes imersivos}

Ambientes imersivos, cada vez mais explorados no campo do entretenimento através das tecnologias, vêm também conquistando espaço no âmbito educacional. Situações de ensino e aprendizagem, calcadas na experiência prática, encontram nos metaversos espaços muito propícios para o seu desenvolvimento, devido ao alto nível de interatividade oferecido pelos mundos virtuais.

A proliferação de soluções para a implantação de mundos virtuais enseja o projeto e implementação de laboratórios virtuais, que possam criar condições para o desenvolvimento de competências e habilidades para interpretar resultados, a partir de experimentos e demonstrações, tal como recomendado nos Parâmetros Curriculares Nacionais (PCN) do MEC (MAYA, 2012). Mas, como bem destaca o documento dos PCN, a experimentação formal em laboratórios didáticos, por si só, não soluciona o 
problema de ensino-aprendizagem. É preciso que, em consonância com as atividades de laboratório, sejam desenvolvidas discussões coletivas levando a reflexões através das quais possam ser construídos conceitos e desenvolvidas competências e habilidades.

Um novo passo nesta proposta pedagógica está na utilização de ambientes imersivos como espaços onde o estudante não somente interage com o objeto de estudo, mas também experiência uma presença virtual que o conduz a uma imersão na situação de ensino-aprendizagem, garantindo maior engajamento nas atividades propostas. Um dos pressupostos da pesquisa foi o princípio de que a interatividade seja importante e relevante para ensejar aprendizagem.

Este princípio tem sido comprovado por inúmeros estudos e em especial foi tornado conhecido, a partir do trabalho de Bloom (1984), que comparou o resultado em termos de aprendizagem de estudantes que receberam atenção como grupo, tal como ocorre em salas de aula, versus resultados alcançados por estudantes que receberam um atendimento individual. Este estudo ficou conhecido como o "2 Sigma Problem", porque a média do desempenho dos estudantes atendidos individualmente por tutores foi 2 desvios padrão $(2 \sigma)$ acima do desempenho dos estudantes que receberam atendimento em grupos de cerca de 30 alunos.

Posteriormente, Fletcher (2001) investigou mais detalhadamente o que acontecia nas duas modalidades de atendimento e constatou que a quantidade de interações entre $o$ professor/tutor e um estudante variava de cerca de 1 interação/hora/estudante na modalidade atendimento em grupo, para cerca de 140 interações entre estudante e tutor/hora. Embora questões de custo sejam impeditivas para que a solução do atendimento individualizado possa ser disseminada, o avanço da tecnologia, em especial no uso de tutores inteligentes, possibilita replicar parcialmente a atenção que um tutor humano proporcionaria ao estudante, quando em atendimento individualizado, e melhorar o resultado da aprendizagem em cerca de 1 desvio padrão, quando comparado com o atendimento em classes de 30 alunos, segundo Fletcher (2001).

Em vista dos potenciais benefícios derivados do uso de sistemas de tutoria inteligentes a pesquisa ora relatada investigou a viabilidade tecnológica para implantação de agentes com algum grau de inteligência para apoiar a atividade dos participantes de experimentos e atividades de aprendizagem em mundos virtuais. A investigação utilizou um ambiente implementado mediante o uso do software livre OpenSim e os agentes foram construídos usando a estratégia de Non Player Character (NPC), tal como descrito em (OpenSim 2013). Para ampliar a flexibilidade das interações entre o estudante, através de seu avatar e o NPC, foi estabelecida uma interconexão deste com um chatterbot cuja base de conhecimento foi preparada para oferecer respostas relevantes ao contexto do experimento realizado.

Segundo Laven (2000) chatterbots são programas de computador que usam inteligência artificial para simular conversas inteligentes com usuários. Laven apresenta uma classificação que considera os recursos utilizados: clássicos (primeiras implementações com estratégias de conversação limitadas), complexos (com personalidades variadas), amigáveis (que simulam personalidades, usam voz e tentam parecer humanos amistosos) e ensináveis (podem adquirir novos conhecimentos a partir da conversação ou de comandos enviados pelo usuário). São também referidas três gerações de chatterbots. 
A primeira geração, baseada em técnicas de casamento de padrão e regras gramaticais, tem como principal ícone ELIZA, um chatterbot desenvolvido no MIT (Artificial Intelligence Lab) por Joseph Weizenbaum (1966), que age como um psicanalista virtual, visando que seu usuário fale cada vez mais de seus problemas através de sua interação com ele, fazendo pergunta se estimulando o desabafo do usuário. Porém, ELIZA não possui memória, logo, não armazena conversas anteriores com os usuários. A segunda geração, baseada em técnicas de inteligência artificial, como as regras de produção e redes neurais é lembrada pelo JULIA, de Michael Mauldin (1994). A terceira e mais recente geração de chatterbots é baseada no uso de linguagens de marcação para a construção de bases de conhecimento. Utilizam a linguagem Artificial Intelligence Markup Language (AIML). Seu maior representante é Artificial Linguistic Internet Computer Entity (ALICE) (WALLACE, 2003).

Existem diversos estudos e experimentos que comprovaram as vantagens na utilização de chatterbots no processo de ensino/aprendizagem destacando: poder de interação, interface agradável, atendimento contínuo em qualquer momento, além de oferecer um dialogo mais personalizado que pode contribuir para motivar e interessar o estudante (Leonhardt (2003), Thiry (2005), Boff (2008), Aguiar (2014)). O trabalho de Batista apresenta um Chatterbot que pode ser usado em Ambientes Virtuais de Aprendizagem (AVAs) para guiar o aluno na obtenção de explicações relacionadas ao FAQ [Batista et. al. 2009]. O AVA INES oferece o CHARLIE, um Chatterbot inteligente, capaz de se comunicar com os alunos em linguagem natural para ajudá-los em suas tarefas e para oferecer conteúdo específico (MIKIC et. al. 2009).

Saceano (2000) classifica os bots em chatterbots (robôs que conversam livremente via teclado, podendo ou não ser multimídia), searchbots (que procuram e filtram Web sites), mailbots (classificam e respondem perguntas via e-mail) e modbots (moderadores de newgroups), além de outros tipos. No presente trabalho foi utilizado um chatterbot baseado na máquina de inferência ALICE de Wallace (2003) e que pode ser usado a partir de um servidor online público Pandorabots (AIMLESS 2004).

\section{Chatterbots em Mundos Virtuais}

Agentes virtuais tem reconhecida utilidade em mundos virtuais $3 \mathrm{D}$, pois a complexidade natural de atuar em um laboratório real não desaparece quando se utiliza laboratórios virtuais. Assim, é preciso proporcionar aos usuários orientação e apoio para que estes possam realizar as atividades previstas. Adicionalmente um agente virtual pode ser delineado para ser usado como ferramenta potencializadora de colaboração, incentivando e requisitando interação com os estudantes que participam simultaneamente da atividade desenvolvida no mundo virtual.

Existem diversas modalidades como um agente virtual pode ser usado em um mundo virtual. Bogo (2003 apud Wooldridge, 2002) conceitua agente como um programa que auxilia o usuário na realização de alguma tarefa ou atividade. Agentes inteligentes tratam-se basicamente de um sistema capaz de tomar decisões e interagir com o ambiente ou outros usuários com base em alguma fonte de dados. (Leonhardt, Tarouco, 2006). Os trabalhos que abordam chatterbots no ensino (Domingues, 2003) normalmente utilizam o chatterbot como um tutor, que faz perguntas ao estudante e provoca respostas por parte dele. 


\section{Non Player Characters}

O mundo virtual é composto por atores que podem estar executando diferentes papéis, como no caso dos estudantes, que manipulam suas representações gráficas (avatares) para realizar diferentes tipos de ações no mundo virtual, como tocar em um objeto, escrever texto e se mover, a partir dos comandos executados por eles. Gascó (2014) explica que além destes atores e papéis existentes em mundo virtual, um outro elemento que é um robô virtual, denominados de bots ou Non Player Characters (NPCs).

Este personagem não jogável/manipulável é um personagem usualmente presente em jogos eletrônicos e embora não possa ser diretamente controlado por um jogador se envolve de alguma forma no enredo de um jogo ou simulação (OLIVEIRA et al., 2012). Desta forma, ele pode exercer um papel específico e interagir com o avatar controlado pelo estudante, com o objetivo de atuar como um ajudante ou guia virtual. Os NPCs podem possuir uma aparência igual ou similar ao avatar controlado pelo estudante. É importante ressaltar também que os NPCs possuem limitações dependendo do ambiente onde estão sendo utilizados. No contexto do OpenSim onde o presente trabalho foi testado, um NPC tem sua atuação limitados a regiões específicas e não podendo se deslocar e atuar em todo o mundo virtual.

O controle de um NPC no ambiente OpenSim é feito por meio de scripts acoplados em objetos de controle executando comandos Linden Scripting Language (LSL) e OpenSim Scripting Language (OSSL) (OpenSim 2013). A ativação de um NPC pode ocorrer por meio de um toque do avatar em algum objeto especialmente criado no mundo virtual. Também pode ser ativado por meio de um comando registrado no chat, assim como por meio de sensores que detectam a presença do avatar e realizam automaticamente a ativação do NPC para interagir com o mesmo.

Por exemplo, assim é possível definir que um NPC irá realizar um conjunto de etapas previamente definidas por meio da linguagem de script OSSL, sendo que no momento de sua ativação, este conjunto de ações será desempenhada pelo bot. Este conjunto de ações pode englobar a sua interação com o avatar por meio do chat, explicando algum tipo de experimento existente no mundo virtual ou responder a questionamentos realizados por algum avatar, tocar algum tipo de objeto, dentre outras atividades.

Além da função de interação com o usuário (via seu próprio avatar) o NPC também pode efetuar um registro da interatividade ocorrida entre ele e os avatares no mundo virtual, sejam por meio de logs do chat ou algum outro tipo de recurso, com o objetivo de proporcionar elementos adicionais para a avaliação das ações do estudante durante o período de atividade no mundo virtual.

\section{Estudo de caso}

O experimento realizado envolveu o uso de um chatterbot no mundo virtual OpenSim para atuar como instrutor e guia do metaverso. O NPC foi interligado ao servidor externo PandoraBots com vistas a ampliar sua capacidade de interação com o usuário. A escolha da plataforma Pandorabots.com para proporcionar a base de apoio do NPC em suas interações derivou da facilidade de criar "pessoas virtuais" neste ambiente.

O Pandorabots.com é um serviço de provedor de um software experimental de robô baseado no trabalho do Dr. Richard Wallace e da comunidade de software livre ALICE/AIML FREE SOFTWARE COMMUNITY (AIMLESS, 2004). Outro fator que 
influenciou a escolha desta plataforma foi o fato de trabalhar com software livre e de código aberto, além da facilidade de acessá-lo.

Usando qualquer browser, é possível a qualquer pessoa criar, modelar e publicar os próprios robôs de software e disponibilizá-los para o público via Internet, registrar gratuitamente e iniciar a criação de seus próprios robôs virtuais. Ele trabalha com a linguagem de especificação da base de conhecimento AIML que permite usar uma base já existente ou criar uma nova base de conhecimento a partir do zero.

O OpenSim é um metaverso também baseado em software livre. Este direcionamento ocorreu não somente pelo acesso livre, independentemente dos objetivos de empresas, que podem sofrer alterações ao longo do tempo, seguindo as necessidades do mercado, mas por abrir possibilidades que contar com a participação das comunidades de software livre, e que permite maior autonomia no uso da ferramenta e, tão logo, maior autonômica sobre o ambiente a ser construído.

\subsection{Conexão do NPC no OpenSim com o ambiente Pandorabots ao OpenSim}

A primeira providência para estabelecer a interligação entre o NPC e Pandorabots é naturalmente a criação de uma conta no "Pandorabots" . O nome e dados de acesso ao bot criado devem ser obtidos, pois serão necessários para a configuração do NPC. O Pandorabot criado deve ser tornado público.

A segunda parte da tarefa envolve a criação de um NPC, ou seja, um personagem programável e não manipulável por um avatar, em mundos virtuais. Desta forma o usuário terá a sensação que esta interagindo com outro avatar, o que lhe proporcionará uma sensação de tutoria constante. No ambiente OpenSim é necessário fazer algumas edições no arquivo OpenSim.ini, que esta na pasta /BIN. Comandos de configuração deverão ser ajustados, tal como ilustrado a seguir:

Algumas atribuições devem ser alteradas

1. Enabled $=$ true na sessão $[\mathrm{NPC}]$;

2. Enabled $=$ true na sessão [XEngine];

3. AllowOSFunctions $=$ true na sessão [XEngine];

4. OSFunctionThreatLevel $=$ VeryHigh na sessão [XEngine].

Será alterado o [XEngine] na seção [GridInfo] e adicionar as seguintes linhas específicas de funções NPC

1. Allow_osNpcCreate $=$ true;

2. Allow_osNpcMoveTo = true;

3. Allow_osNpcRemove = true;

4. Allow_osNpcSay = true;

5. Allow_osAvatarPlayAnimation = true;

6. Allow_osAvatarStopAnimation $=$ true .

Feito isso o arquivo OpenSim.ini deverá ser salvo. Cabe destacar que tais alterações afetarão todas as regiões. A seguir devem ser editados os scripts em um prim

\footnotetext{
${ }^{1}$ Disponível em: < http:/pandorabots.com >.
} 
(objeto do mundo virtual) disparador do NPC. Quando este objeto for tocado pelo avatar será instanciado um NPC. A programação do NPC para a comunicação com o chatterbot do Pandorabot envolve o uso de comandos tal como no exemplo seguinte:

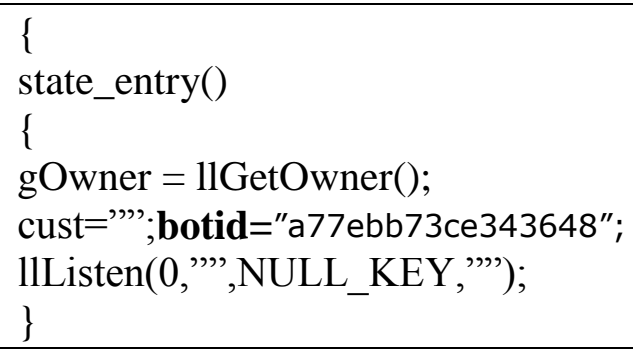

O código que estabelece o link com o banco de dados do Pandorabots usa comandos como nos exemplos seguinte:

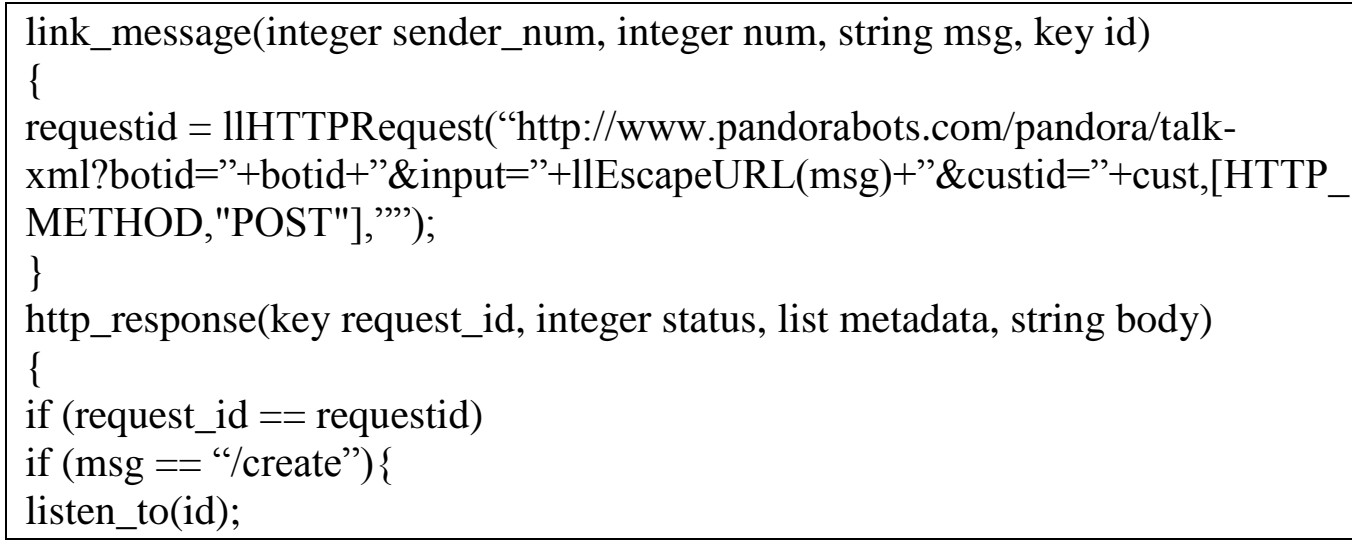

A criação do NPC com numero UUID do avatar, é feita usando o código:

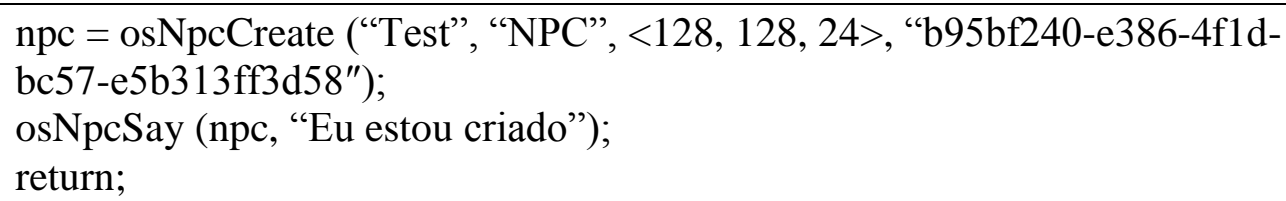

Pode-se adicionar movimentação do NPC, com códigos tal como os abaixo

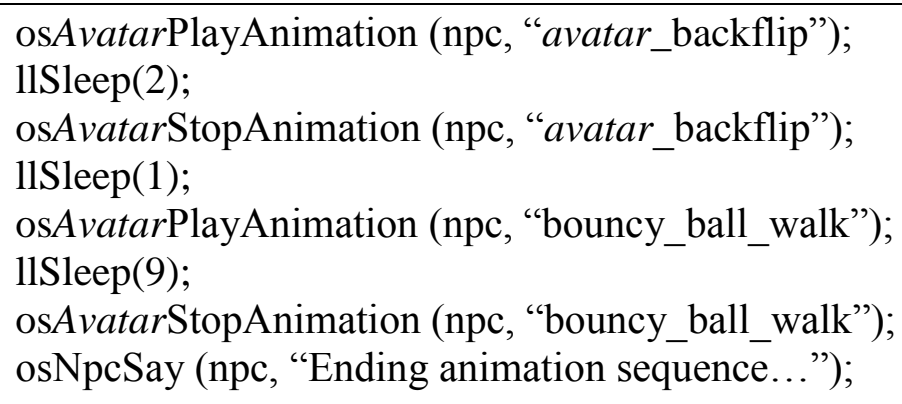

Com tais codificações é possível instanciar um NPC, ligado a base de dados do Pandorabots. A base de dados do chatterbot pode ser personalizada para o experimento onde o chatterbot vai intervir. A edição da base de conhecimento do chatterbot é feita editando um arquivo construído com a linguagem de marcação AIML. As principais tags do AIML são: 
<aiml> inicia e termina um bloco programado em AIML;

$<$ category> identifica uma "unidade de conhecimento" na base de conhecimento;

$<$ pattern> identifica um padrão de mensagem simples frequentemente utilizado; por usuários;

$<$ template> contém a resposta para uma mensagem do usuário.

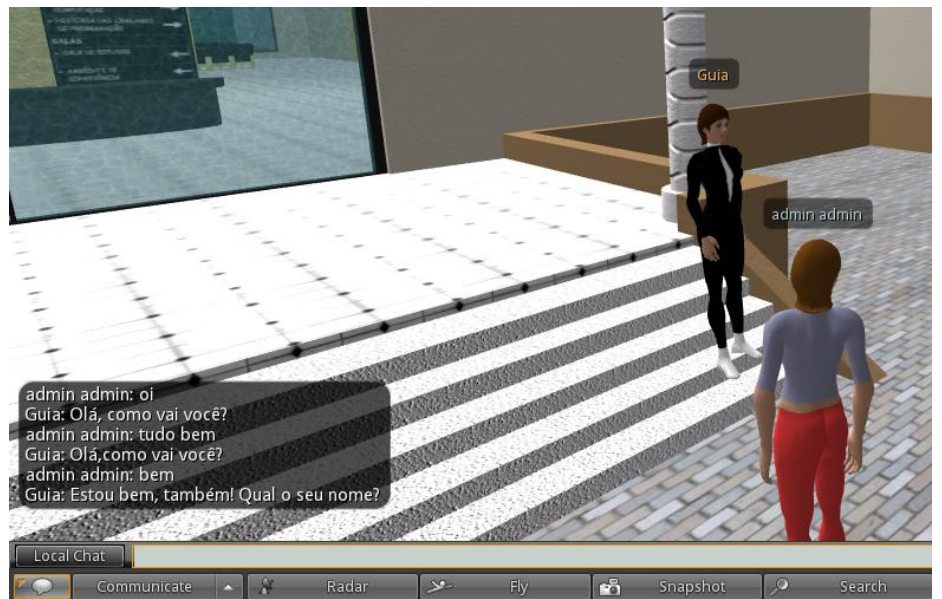

Figura 1. Chatterbot do Pandora integrado com o OpenSim

Neste exemplo visto na Figura 1, o padrão de entrada "Oi" seguido de qualquer informação, terá como resposta ou possível resposta: "Olá como vai você?"; "Tudo bem"; "Estou bem também, qual é seu nome?". Com a utilização de AIML, pode-se definir mais de uma resposta para um padrão e ainda se podem especificar critérios de escolha de cada uma das respostas que a base de conhecimento pode conter.

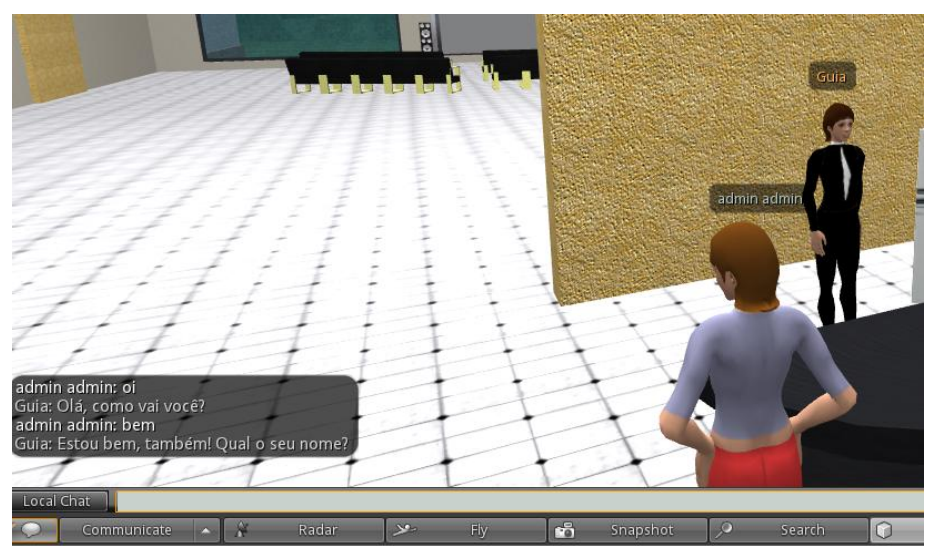

Figura 2. Guia tutor interagindo com o aluno

$\mathrm{Na}$ Figura 2, o exemplo mostra o guia tutor interagindo usando as interações sociais (conhecimento genérico usável em qualquer diálogo usuário- chatterbot), antes de responder sobre a sua base de conhecimento específico do contexto onde esteja sendo usada. 


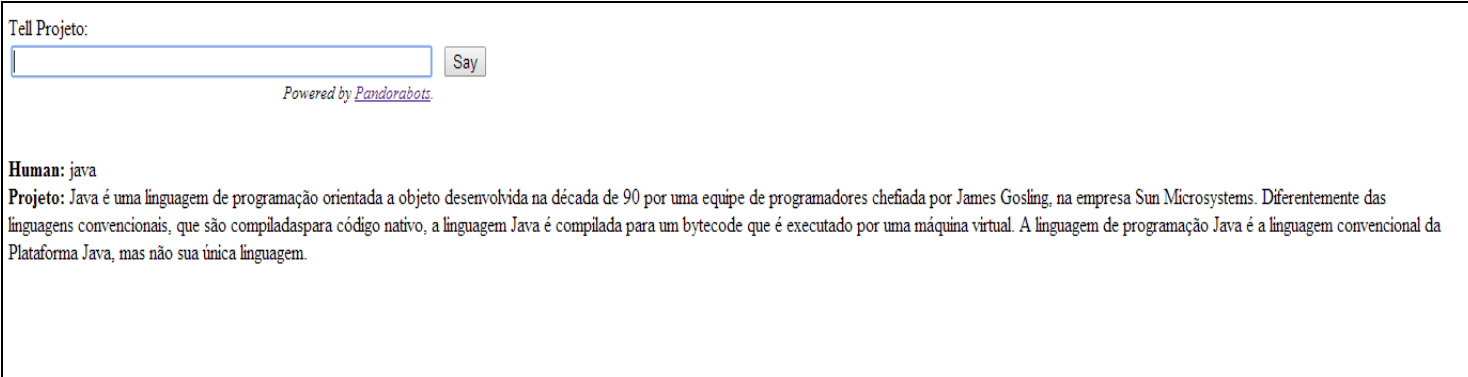

Figura 3. Página do site do Chatterbot Pandora

Na Figura 3 é mostrado como o chatterbot mostra a sua base de conhecimento especifica, falando sobre os objetos e artefatos encontrados no mundo virtual. O avatar aluno é representado como human e o chatterbot se apresenta como projeto respondendo a pergunta buscada na sua base de conhecimento especifica, ou seja, ao aluno digitar "Java", ele buscou sobre a linguagem de programação Java.

\subsection{Interação dos usuários de mundos virtuais e chatterbot}

Embora existam diversas pesquisas envolvendo a interligação de chatterbots em ambientes virtuais, o uso do tipo de interligação testada no presente experimento não é comum. A presente pesquisa envolveu, além da implementação de um protótipo, também um teste de aceitação e impacto nos usuários visando investigar questões tais como:

- os usuários de mundos virtuais querem interagir com um chatterbot guia tutor?

- os usuários utilizariam esse tipo de recurso?

De acordo com os resultados obtidos, a resposta a essas duas perguntas é sim, o que aponta para benefícios relevantes derivados da integração de chatterbots com mundos virtuais tal como testado no presente caso, usando o OpenSim e o ambiente Pandorabots. Além de responder as duas perguntas, a avaliação de viabilidade também trouxe resultados que confirmam que este tipo de solução atende às expectativas dos usuários em termos de suporte esperado para facilitar e orientar quanto ao uso dos recursos disponíveis em um mundo virtual.

Muitas opções se abrem com esta perspectiva tal como a possibilidade do uso de chatterbots no ensino, que tal como destacado por Wazlawick (2001), pode auxiliar no processo de construção do conhecimento dos estudantes, a partir de uma posição mais ativa (raciocínio para a formulação das perguntas a serem feitas) e não pela simples resposta às perguntas do tutor.

\section{Considerações Finais}

A evolução ocorrida nas últimas décadas com relação aos mundos virtuais tem permitido que o uso dos seus recursos para fins educacionais. Um exemplo disso é a criação de NPCs neste ambiente, que possibilitam a interação destes com os avatares controlados pelos usuários, de forma individualizada ou conjunta.

Este artigo apresentou um relato de pesquisa acerca da criação de NPCs no mundo virtual, detalhando todo o seu processo de desenvolvimento e a sua interconexão com uma base de dados inteligente. Assim, foi demonstrado como realizar todo 
processo de criação de um NPC no mundo virtual OpenSim, de forma que este esteja interconectado à base de dados do Pandorabots.

Com isso, foi demonstrado que uma aprendizagem individualizada, com possibilidades ampliadas de interação pode ser construída em mundo virtual, no qual os usuários por meio de seus avatares irão interagir com os NPCs de acordo com as suas necessidades e tarefas dispostas, fornecendo uma nova possibilidade de abordagem educacional. Esta maior interação visa melhorar o desempenho dos estudantes tal como demonstrado por Fletcher (2001) como forma de tratar o "2 Sigma Problem" elicitado por Bloom (1984).

Este trabalho constituiu o início de uma linha de investigação envolvendo a criação de outros laboratórios virtuais e agentes virtuais especialmente construídos para orientar a atividade no laboratório e instigar reflexões sobre as ações e resultados obtidos.

\section{Referências}

Aguiar, E. V .B., Tarouco, L. R. T., Reategui, E. (2014) "Supporting Problem-Solving in Mathematics with a Conversational Agent Capable of Representing Gifted Students' Knowledge", In: Annual Hawaii International Conference on System Sciences - HICSS-47 Waikiloa, USA 201447 130-137.

Aimless, D \& Umatani, S. (2004) “A Tutorial for adding knowledge to your robot". Disponível em: http://www.pandorabots.com/botmaster/en/tutorial?ch=1 Acessado em 19/07/2014

ALICE. A.L.I.C.E Artificial Intelligence Foundation. Acessível em http://alice.pandorabots.com/. Acessado em Junho de 2012.

Barbosa, S.D. J; Silva, B.S. (2010). "Interação Humano- Computador”, In: Editora Campus.

Batista, A. M.; Marietto, M. G. B.; Barbosa, G. O. Franca, R. S.; Kobayashi, G. (2009) "Multi-agent systems to build a computational middleware: A chatterbot case study", In: International Conference for Internet Technology and Secured Transactions (ICITST 2009), pp. 1-2.

Bloom, B. (1984)."The 2 Sigma Problem: The Search for Methods of Group Instruction as Effective as One-to-One Tutoring", Educational Researcher, 13:6 (4-16).

Boff, Elisa. (2008) "Colaboração em Ambientes Inteligentes de Aprendizagem mediada por um Agente Social Probálistico”, In: Tese - PPGC, UFRGS. Porto Alegre.

Bogo, Luís Henrique (2003) "Criação de Comunidades Virtuais a partir de Agentes Inteligentes: Uma aplicação em E-learning", In: Dissertação (Mestrado em Engenharia de Produção) - PPGEP, UFSC. Florianópolis.

Domingues, Maria J. C. de S. (2003) "Mídia e Aprendizagem: Um estudo comparativo entre Hipertexto e Chatterboot", In: Tese de Doutorado, UFSC.

Fletcher, J. D. (2001) "Technology, the Columbus Effect, and the Third Revolution in Learning", In: IDA Document. Institute for Defense Analyses. p. 32.

Gascó, M., Rubio, R., Sancho, S., Martínez, V. (2014) "EDUCATIVE 3D VIRTUAL ENVIRONMENTS”, In: INTED2014 Proceedings, pp. 5525-5529. 
Laven, S. "The Simon Lavel Homepage". Disponível em: http://www.simonlaven.com/ Acesso em: 17 jul 2014.

Leaverton, M. “Tech Trends Homepage". Disponível em: http://www.alicebot.org/press. Acesso em: 17 jul 2014.

Leonhardt, N., Neisse, R., Tarouco., L. (2013) "MEARA: Um Chatterbot Temático para Uso em Ambiente Educacional”, In: XIV Simpósio Brasileiro de Informática na Educação (SBIE 2003) NCE - IM / UFRJ.

Leonhardt, M., Castro, D., Dutra, R., Tarouco, L. (2003) "ELEKTRA: Um Chatterbot para Uso em Ambiente Educacional”, In: RENOTE. 2V1 N2 CINTED/UFRGS. $1-10$.

Leonhardt, M.; Tarouco. L. "Doroty: um chatterbot para aprendizado baseado em problemas aplicado ao treinamento de profissionais de gerência de redes". XVII Simpósio Brasileiro de Informática na Educação (SBIE 2006) UNB/UCB.

Mauldin, Michael (1994), "Chatterbots, TinyMuds, and the Turing Test: Entering the Loebner Prize Competition", In: Proceedings of the Eleventh National Conference on Artificial Intelligence, AAAI Press.

Maya, E. "Parâmetros Curriculares Nacionais para o ensino médio". In: MEC.

Mikic, F.; Burguillo, J.; Llamas, M.; Rodriguez, D.; Rodriguez, E (2009). Charlie: An AIML-based chatterbot which works as an interface among INES and humans. In: 39th IEEE Frontiers in Education Conference, pp. 1-6.

OpenSimulator, Non Player Characters (2013). Disponível em http://OpenSimulator.org/wiki/NPC. Acesso em 30 abril 2014.

Saceano, Daniel. "Chatterbots, Nanny-bots e outras criaturas". Disponível em: http://www.suite101.com/article.cfm/future_technology/6411 Acesso em: 19 maio 2014.

Thiry, M., Rios, D. "Um chatterbot para auxiliar no aprendizado em qualidade de software”, In: XI Congresso Argentino de Ciências de la Computación.

Oliveira, P. P. B. (2012) “Ferneda, E.; Prado,H.A;Bittencout,I.I.”Integrando sistemas tutores inteligentes a jogos”, In: 23 Simpósio Brasileiro de Informática na Educação (SBIE 2012).

Wallace, R., Tomabechi, H., Aimless, D., "Chatterbots Go Native: Considerations for an eco-system fostering the development of artificial life forms in a human world by. Alice Foundation". Disponível em http://www.pandorabots.com/pandora/pics/chatterbotsgonative.doc Acesso em 4 abril 2014.

Wazlawick, Raul S. (2001) "Aprendizagem pela criação de museus virtuais interativos; proposta e construção de uma nova ferramenta de autoria em realidade virtual", In: Relatório técnico-parcial do Projeto CNPq-ProTem-CC Museu Virtual, UFSC, 2001.

Weizenbaum, Joseph. (1966) "ELIZA - A Computer Program For the Study of Natural Language Communication Between Man and Machine", In: Communication of ACM, V9, N1, p. 36-45. 